\title{
A Novel Unidirectional Porous $\beta$-tricalcium Phosphate Bone Substitute in Orthopedic Surgery: A Technical Note and Case Illustrations
}

\author{
Toru Funayama $^{1}$, Hiroshi Noguchi ${ }^{1}$, Hiroshi Kumagai ${ }^{1}$, Tomokazu Yoshioka ${ }^{1}$, Masashi Yamazaki ${ }^{1}$
}

1. Orthopaedic Surgery, University of Tsukuba, Tsukuba, JPN

Corresponding author: Toru Funayama, funatoru3@gmail.com

\begin{abstract}
Bone grafting is frequently performed in orthopedic surgeries. Artificial bone is a common grafting material. We have developed an absorbable material for bone regeneration with a unique structure: unidirectional porous $\beta$-tricalcium phosphate (Affinos ${ }^{\circledR}$; Kuraray Co., Ltd., Tokyo, Japan). The most distinctive feature of this material is the ease with which blood can rapidly reach its depths by capillary action due to the unidirectional porous structure. It is also characterized by the presence of micropores, which are known to be beneficial for osteoconductivity both on the surface and inside the material. Favorable artificial bone absorption and regeneration with natural bone were observed in cases of clinical bone graft applications in the spine, extremities, benign bone tumor, trauma, and donor sites. Affinos is useful as a novel absorbable material for bone regeneration in various orthopedic surgeries.
\end{abstract}

Categories: Orthopedics

Keywords: $\beta$-tricalcium phosphate, orthopedic surgery, bone graft, artificial bone, unidirectional porous structure

\section{Introduction}

Bone grafting is frequently used in orthopedic surgery [1]. The commonly used types of bone grafts vary from country to country. In Japan, these are autologous (56.4\%), artificial (40\%), and allogenic bone grafts (3.6\%) [2]. Allogenic bone grafting is used in some cases, mainly for the supplementation of large bone defects, such as in revision total hip arthroplasty in Japan, and the trend remains unchanged these days [3]. Only a limited amount and quality of autologous bone can be harvested from osteoporotic patients, whose numbers have been increasing due to the rapid aging of the population in recent years. Therefore, artificial bones are largely used to meet the needs of bone grafting during orthopedic surgery.

\section{Technical Report}

Received 03/16/2020

Review began 03/19/2020 Review ended 03/26/2020 Published 03/30/2020

() Copyright 2020

Funayama et al. This is an open access article distributed under the terms of the Creative Commons Attribution License CC-BY 4.0., which permits unrestricted use, distribution, and reproduction in any medium, provided the original author and source are credited.
We have developed a unidirectional porous $\beta$-tricalcium phosphate $(\beta-\mathrm{TCP})$ material, Affinos ${ }^{\circledR}$ (Kuraray Co., Ltd., Tokyo, Japan) for use as an artificial bone with a unidirectional porous structure in which efficient tissue penetration and high osteoconductivity can be expected (Figure 1). 


\section{Cureus}

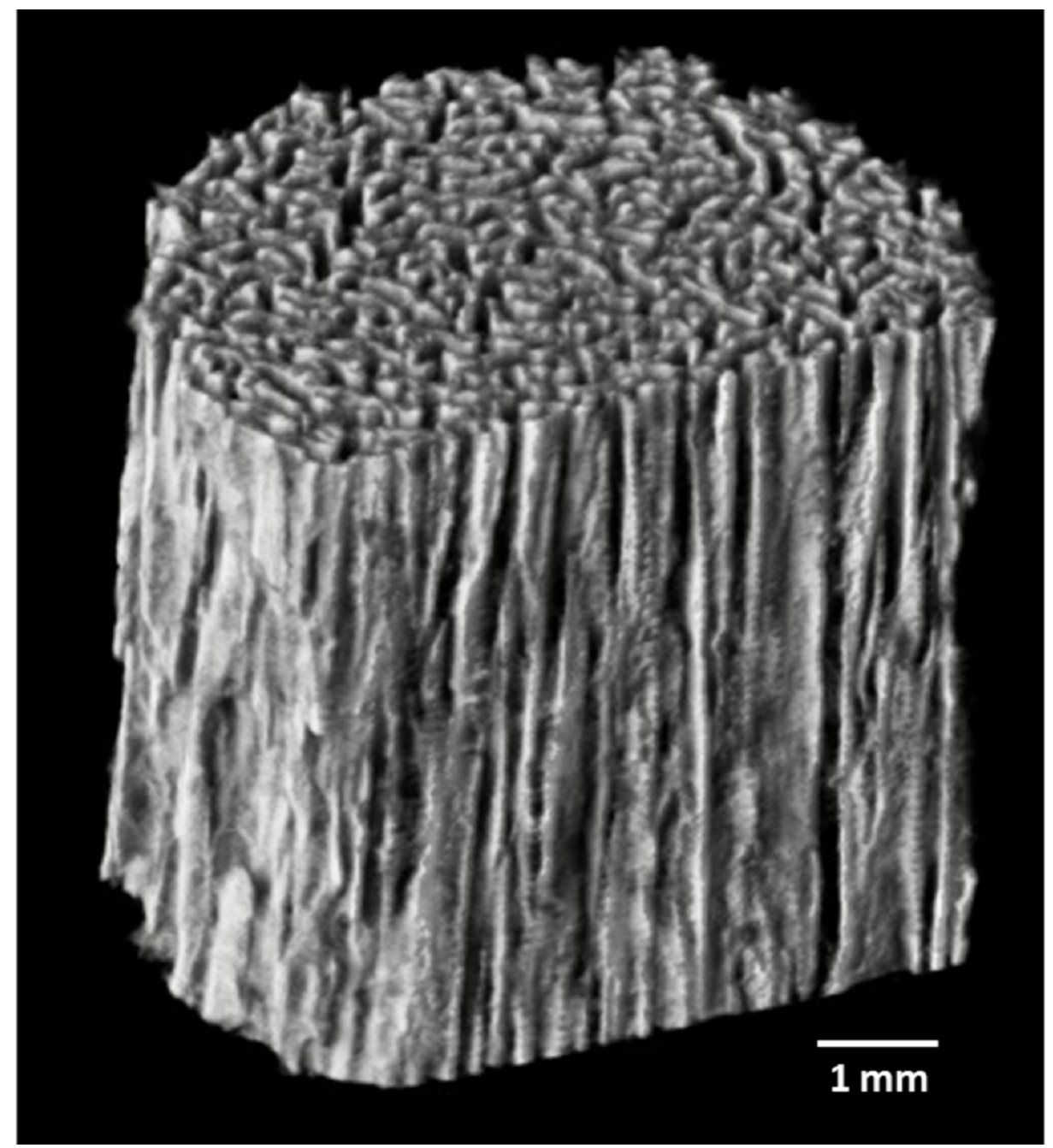

\section{FIGURE 1: 3D-CT image of Affinos}

Affinos has a unique, characteristic structure in which unidirectional pores 25-300 $\mu \mathrm{m}$ in diameter are aligned in one direction.

Affinos: Kuraray Co., Ltd., Tokyo, Japan

The porosity of the material is $57 \%$ and the initial compressive strength is $8 \mathrm{MPa}$ and $1.5 \mathrm{MPa}$ in directions parallel and perpendicular to the pores, respectively [4]. The most distinctive feature of Affinos is that blood can reach the depths of the material rapidly by capillary action due to its unidirectional porous structure. It is also characterized by the presence of micropores, which are known to be beneficial for osteoconductivity, both on the surface and inside the material [4]. In tibial defect implant studies in rabbits, balanced resorption and regeneration with autologous bone were observed from an early stage of implantation; new bone and vascularization, as well as the presence of blood flow inside the material, were also observed [4-6]. Furthermore, in another femur defect implant study in a beagle, the porous structure of the material was filled by new bone within three months (Figure 2). 


\section{Cureus}
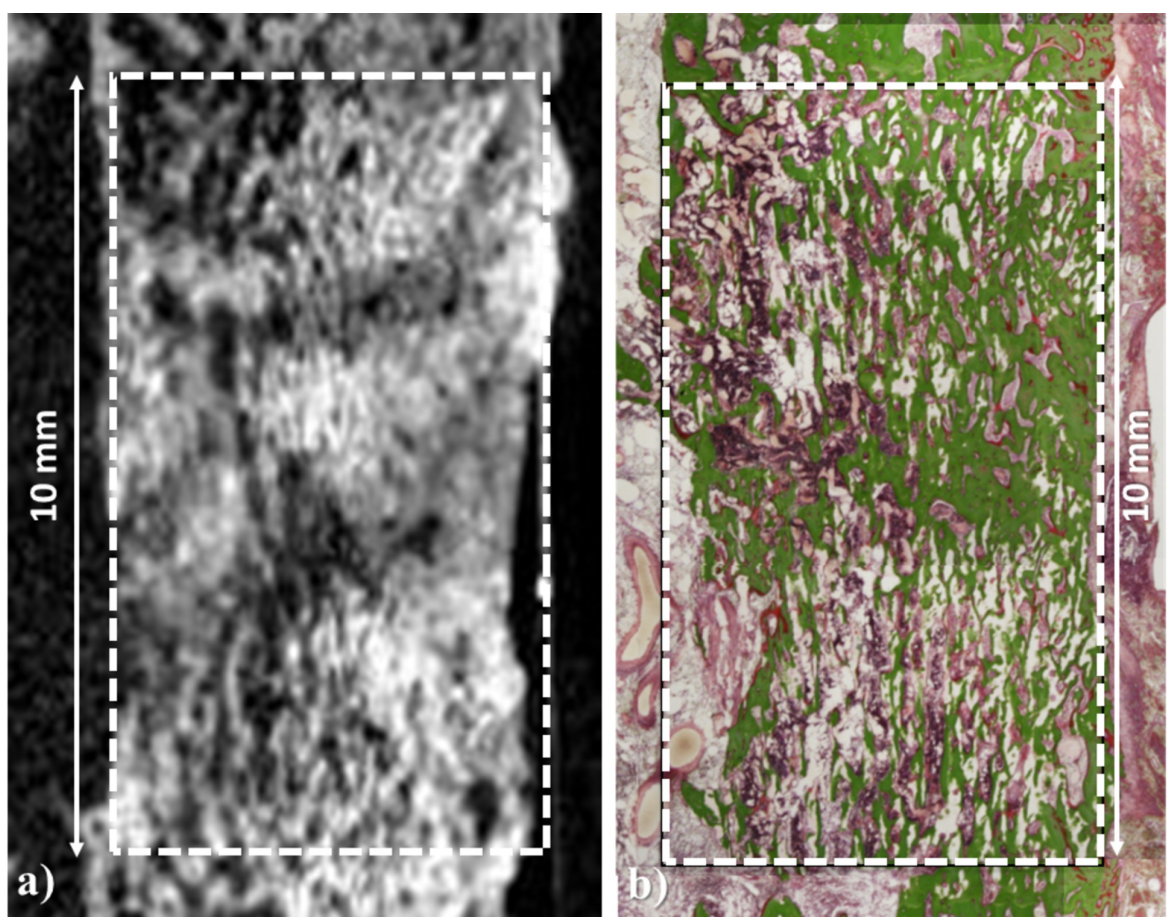

\section{FIGURE 2: An implant experiment in a beagle model of a partial femur}

defect

Ten-millimeter Affinos blocks were implanted into a partial cortical defect prepared in the femoral shaft in which the unidirectional porous direction was matched with the longitudinal axis of the femur. The bone defect was cross-bridged by new bone whose orientation matched the porous structure within three months.

a: Micro CT; b: Villanueva Goldner staining

Affinos: Kuraray Co., Ltd., Tokyo, Japan

Affinos is also expected to present balanced absorption and regeneration similar to autologous bone from an early stage in clinical applications. However, limited clinical application studies have been reported so far [7-13]. Here, we report the benefits of Affinos as an absorbable material for bone regeneration by introducing clinical application cases in various fields of orthopedic surgery. We obtained consent to publish data from all the patients involved.

\section{Case illustration 1 (spine)}

Posterior fusion was performed for a seven-year-old girl with chronic atlantoaxial rotatory fixation. In the procedure, the autologous iliac cancellous bone and Affinos granules were mixed in approximately 1:1 ratio to form a bone graft that filled the space between the occipital bone and the spinous process of the axis. At six months after surgery, favorable fusion and regeneration with natural bone were achieved and at 12 months post-surgery, completion through sufficient remodeling was achieved and the area from the occipital bone to the axis was completely fused (Figure 3). 


\section{Cureus}

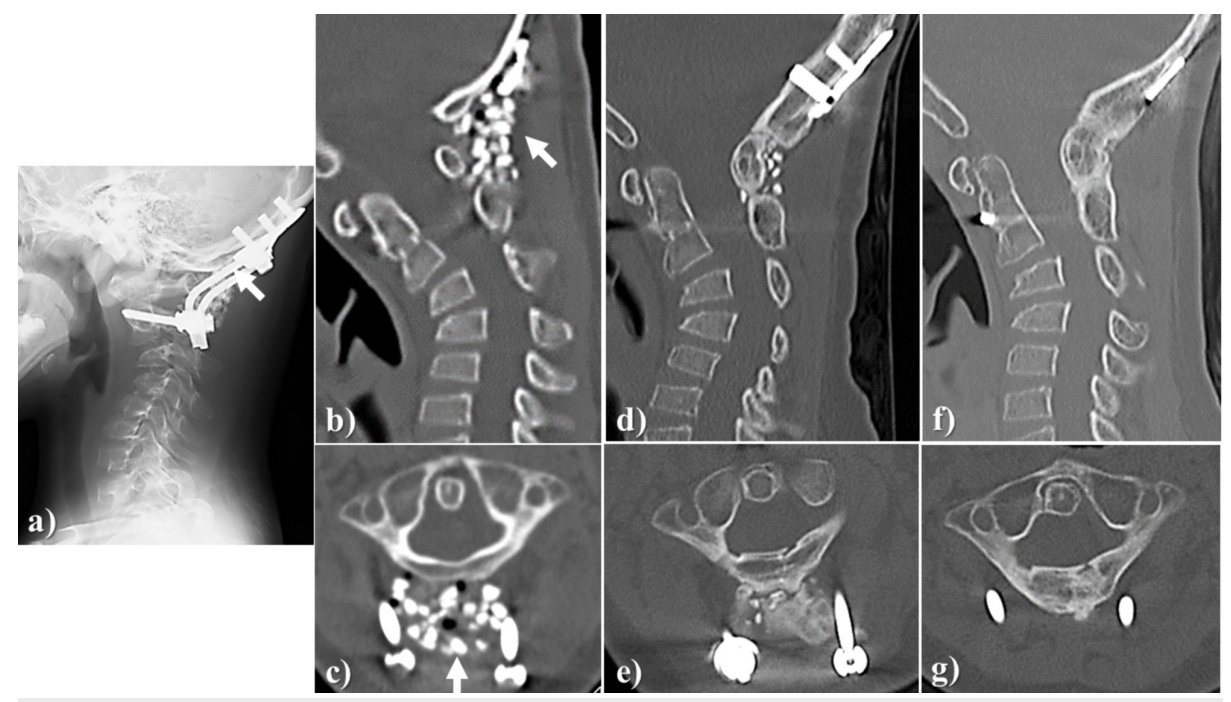

FIGURE 3: Case illustration 1

Posterior fusion was performed for a seven-year-old girl with chronic atlantoaxial rotatory fixation.

a, b, c: The autologous ilium (the cancellous bone) and Affinos granules were mixed in approximately 1:1 ratio to fill the space between the occipital bone and the spinous process of the axis (arrow); d, e: At six months after surgery, favorable bone fusion and replacement with own bone were achieved; $f, g$ : At 12 months after surgery, completion through sufficient remodeling was achieved.

Affinos: Kuraray Co., Ltd., Tokyo, Japan

\section{Case illustration 2 (spine)}

Anterior-posterior fusion was performed for a 74-year-old woman with advanced kyphotic deformity due to vertebral fracture at the upper end of the fusion and adjacent segmental disease after lumbosacral posterior fusion. The autologous iliac bone chips and Affinos granules were mixed in approximately 1:1 ratio to form a bone graft that filled the bone defect generated by resecting the pedicle of the fractured vertebra on the extension of the posterior fusion for kyphosis correction. At three months after surgery, artificial bone resorption and new bone formation progressed. The bone was regenerated as a new pedicle 12 months after surgery (Figure 4).
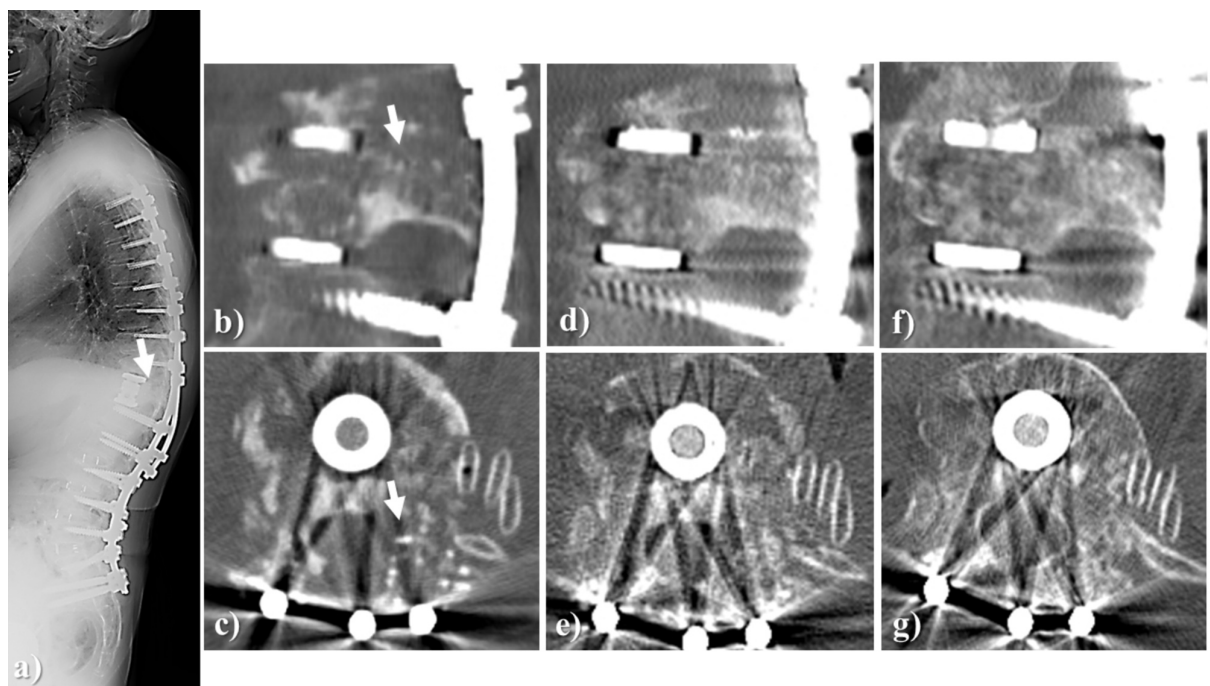

\section{FIGURE 4: Case illustration 2}

An anterior-posterior fusion was performed for a 74-year-old woman with advanced kyphotic deformity due to vertebral fracture at the upper end of the fusion and adjacent segmental disease after lumbosacral posterior fusion.

a, b, c: X-ray of the whole spine and CT images of the left T12 pedicle immediately after surgery. The autologous iliac bone chips and Affinos granules were mixed in approximately $1: 1$ ratio to fill the bone defect 


\section{Cureus}

generated by removing the pedicle of the fractured vertebra (arrow); d, e: At three months after surgery, artificial bone resorption and own bone formation progressed; f, g: At 12 months after surgery, the bone was regenerated as a new pedicle.

Affinos: Kuraray Co., Ltd., Tokyo, Japan

\section{Case illustration 3 (foot)}

A 77-year-old woman with a pes planovalgus deformity underwent a lateral column-lengthening procedure. The bony defect in the surgery was filled with Affinos blocks and granules. At three months after surgery, artificial bone resorption, as well as new bone formation, progressed and at 12 months after surgery, there was complete regeneration with an autologous bone (Figure 5).
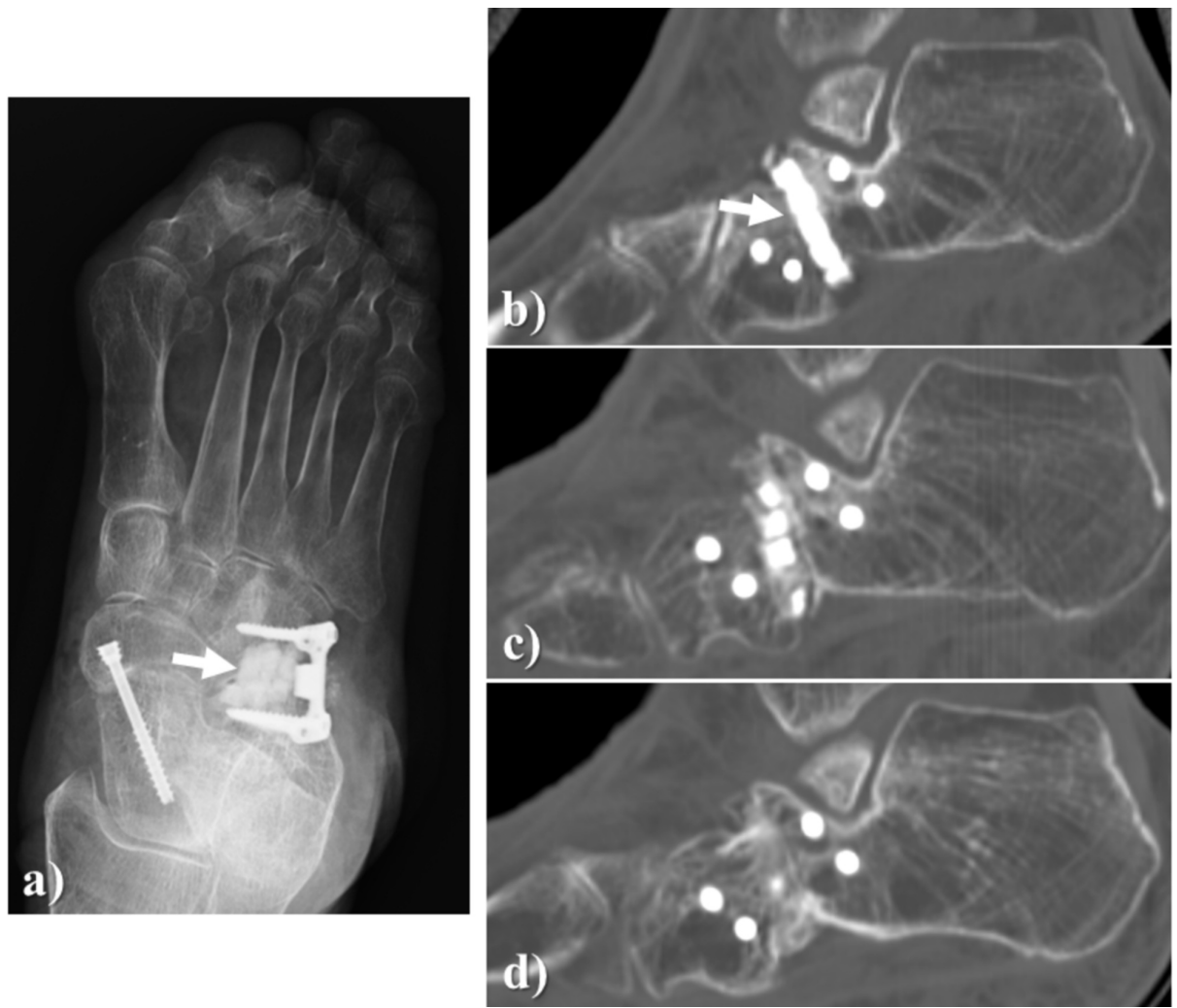

\section{FIGURE 5: Case illustration 3}

A 77-year-old woman with pes planovalgus deformity underwent a lateral column lengthening.

a, b: The bony defect in the surgery was filled with Affinos blocks and granules (arrow); c: At three months after surgery, artificial bone resorption as well as own bone formation progressed; d: At 12 months after surgery, there was complete replacement with own bone.

Affinos: Kuraray Co., Ltd., Tokyo, Japan

\section{Case illustration 4 (benign bone tumor)}

A 16-year-old man had a large bone defect originating from tumor curettage for chondroblastoma of the scapula filled with Affinos. Despite the large number of granules being used $(20 \mathrm{~g})$, artificial bone resorption on the marginal area was observed 3 months after surgery. Moreover, it was observed that the bone graft was clearly replaced with the patient's own bone over time (Figure o). 


\section{Cureus}
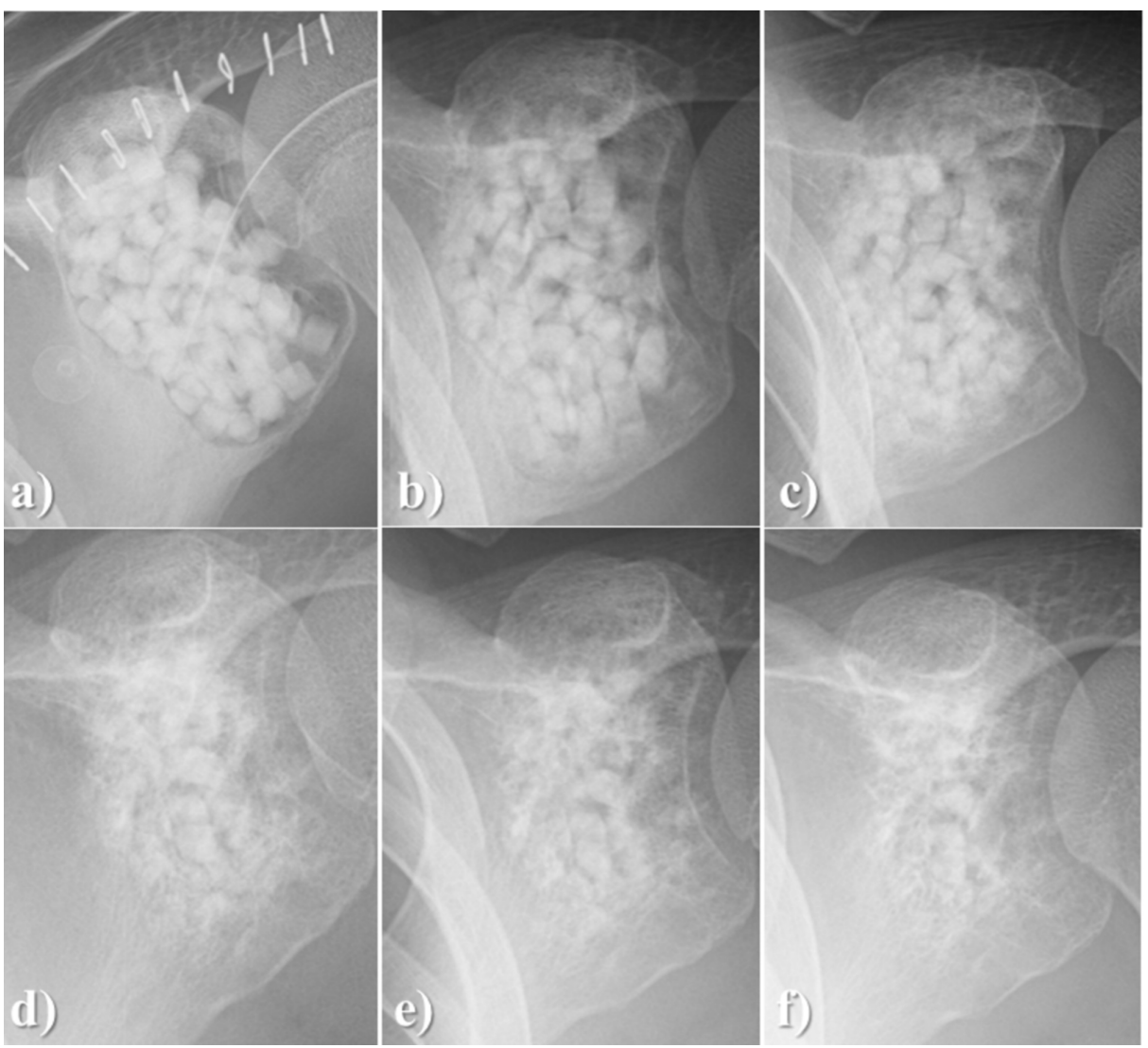

\section{FIGURE 6: Case illustration 4}

A 16-year-old man had a large bone defect originating from tumor curettage for chondroblastoma of the scapula.

a: The bone defect was filled with Affinos granules (total weight, $20 \mathrm{~g}$ ); b: At three months after surgery, artificial bone resorption from the marginal area was observed; c: At six months; d: At 12 months; e: at 18

months; f: at 24 months after surgery, the artificial bone graft was clearly replaced with the patient's own bone

Affinos: Kuraray Co., Ltd., Tokyo, Japan

\section{Case illustration 5 (trauma)}

During surgery for a supracondylar femur fracture, a large bone defect in a 77-year-old woman was filled by large amounts of Affinos blocks and granules (a total of approximately $25 \mathrm{~g}$ ). At three months after surgery, resorption in the marginal area of the artificial bone block was observed and at 6six months after surgery, regeneration with autologous bone in the marginal area was observed. After boney fusion at the fracture site was completed, a state of gradual regeneration with natural bone over time (12 months and 24 months after surgery) was observed (Figure 7).
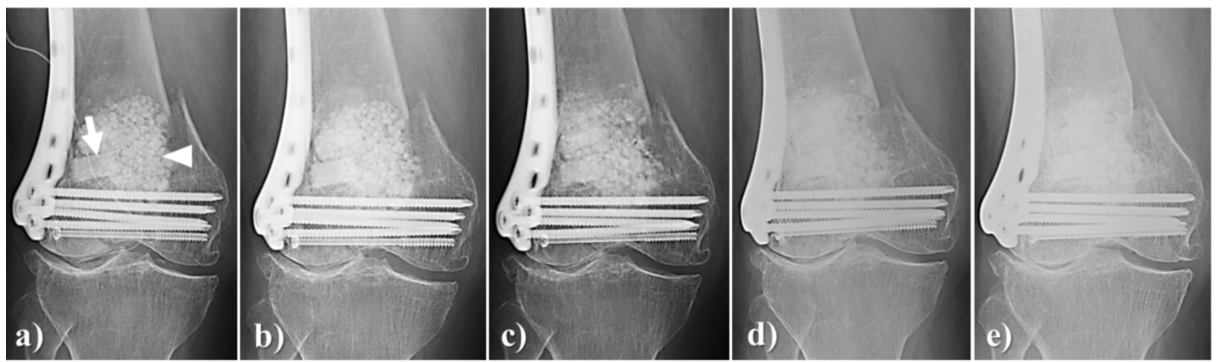

\section{FIGURE 7: Case illustration 5}

During surgery for supracondylar femur fracture, a large bone defect was observed in a 77-year-old woman.

a: Large amounts of Affinos blocks (arrow) and granules (arrowhead) were used (total weight, approximately $25 \mathrm{~g}$ ); b: At three months after surgery, resorption in the marginal area of the artificial bone block was 


\section{Cureus}

\section{Case illustration 6 (bone graft donor site)}

A 22-year-old woman had her fibula harvested and transplanted into the cervical spine for a $7.5 \mathrm{~cm}$ long defect. Affinos cylinder blocks with matched longitudinal and unidirectional pore directions were used. At six months after surgery, fusion was observed with new bone growing downward from the edge of the stump. At 24 months after surgery, the area proximal to the stump was completely regenerated with natural bone and some regeneration in the original tubular structure of the fibula was also seen (Figure 8).
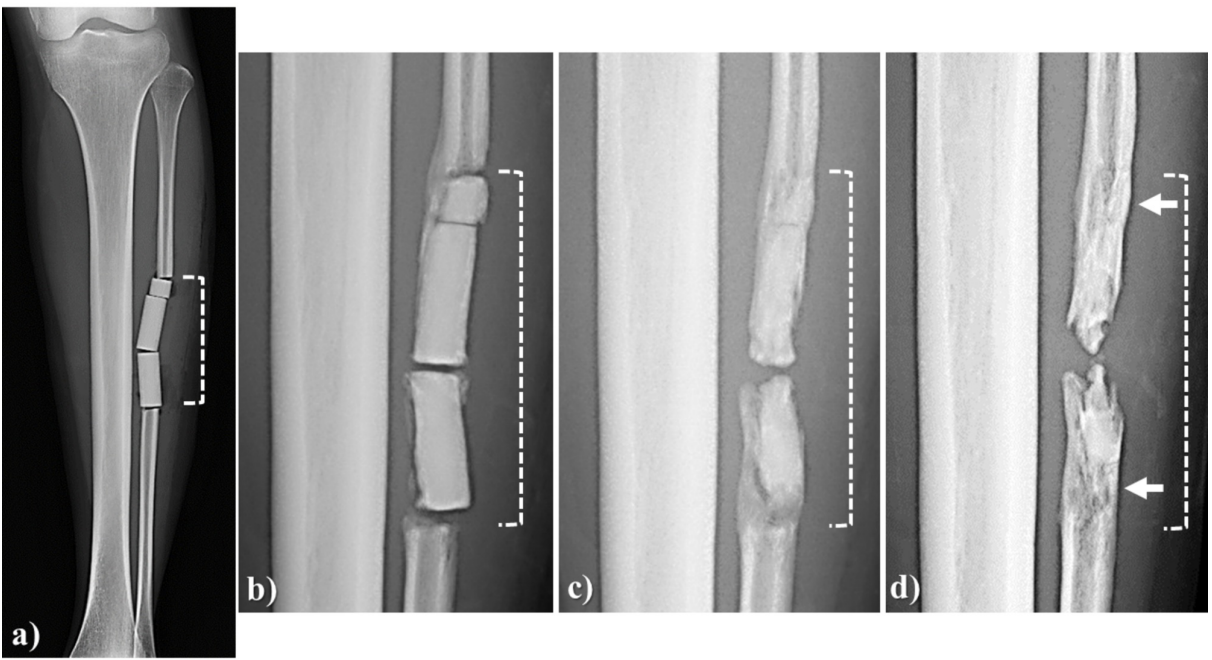

\section{FIGURE 8: Case illustration 6}

A 22-year-old woman had her fibula harvested and transplanted into the cervical spine for a $7.5 \mathrm{~cm}$ long defect (area indicated with a broken line).

a: Affinos cylinder blocks were used in which the longitudinal and unidirectional pore directions were matched; b: At six months after surgery, fusion was achieved by new bone continuing from the stump of the fibula; c: At 12 months after surgery, fusion between blocks progressed and the area close to the stump was replaced with own bone; d: At 24 months after surgery, there was regeneration through the original tubular structure of the fibula in the area close to the stump (arrow).

Affinos: Kuraray Co., Ltd., Tokyo, Japan

\section{Case illustration 7 (bone graft donor site)}

The cancellous bone of the distal radius, in a 16-year-old man, was transplanted to the nonunion area at surgery after a scaphoid fracture. Affinos granules were used for the bone defect at the donor site. At three months after surgery, artificial bone resorption, as well as replacement with own bone, progressed and at 12 months after surgery, the bone graft was completely regenerated with natural bone (Figure 9).
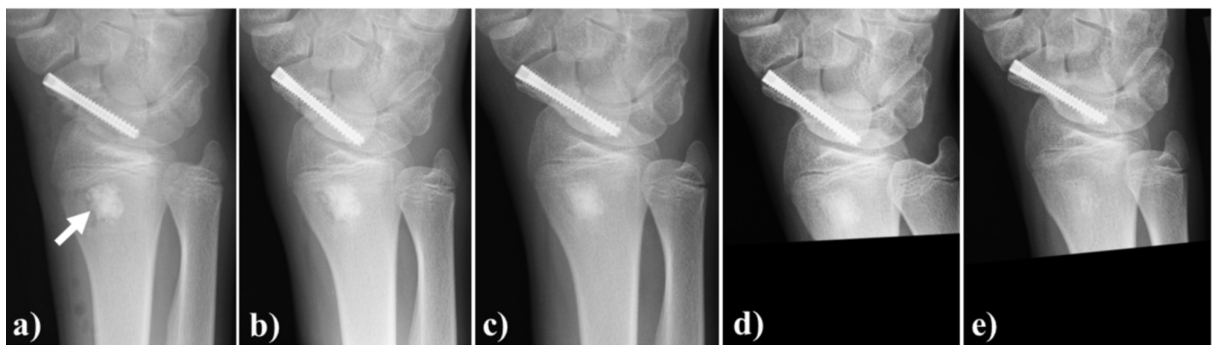

FIGURE 9: Case illustration 7

The cancellous bone of the distal radius in a 16-year-old man was transplanted to the nonunion after a scaphoid fracture. 
a: Affinos granules were used for the bone defect at the donor site (arrow); b: At one month after surgery, artificial bone resorption in the marginal area started; c: At three months after surgery, artificial bone resorption and replacement with own bone progressed; d: At six months after surgery, replacement with own bone progressed further; e: At 12 months after surgery, the bone graft was completely replaced with own bone and trabecular bone structure was regenerated

Affinos: Kuraray Co., Ltd., Tokyo, Japan

\section{Discussion}

Artificial bone is grafted to the cancellous bone area in the host bone in many conditions, such as in full bone defects, vertebral interbody fusions, tumor curettage areas, or nonunion areas, all of which have varying defect sizes and environments. The blood flow and local bone metabolism in the area to which an artificial bone is grafted depends on the diseased condition. For the clinical application cases of Affinos shown in this report, although the speeds of resorption and replacement with own bone varied, replacement with own bone and fusion were certainly achieved in all the cases and thus Affinos is an advantageous material for bone regeneration.

In order to further enhance the remarkable functions of Affinos as a resorbable artificial bone material, we have focused on its integration with teriparatide, bone marrow-derived cells, and platelet-rich plasma. We observed that new bone formation inside the material is promoted by teriparatide administration in an animal experiment [14]. Since Affinos allows bone marrow blood and platelet-rich plasma to rapidly penetrate material in depth by capillary action, these may contribute to the promotion of new bone formation inside the material [4,13]. Thus, Affinos is expected to become a novel absorbable biomaterial with beneficial features for bone regeneration in orthopedic surgery.

\section{Conclusions}

Unidirectional porous $\beta$-TCP (Affinos) is a novel absorbable material for bone regeneration with a unique structure. It is useful in bone grafting in various orthopedic surgeries.

\section{Additional Information \\ Disclosures}

Human subjects: Consent was obtained by all participants in this study. Animal subjects: Laboratory Animal Resource Center of University of Tsukuba Issued protocol number 14-70. Conflicts of interest: In compliance with the ICMJE uniform disclosure form, all authors declare the following: Payment/services info: This study was conducted in collaboration with Kuraray Co., Ltd., Tokyo, Japan. Financial relationships: Toru Funayama and Masashi Yamazaki declare(s) a grant from Kuraray Co., Ltd. This study was conducted in collaboration with Kuraray Co., Ltd. Other relationships: All authors have declared that there are no other relationships or activities that could appear to have influenced the submitted work.

\section{Acknowledgements}

We express our sincere gratitude to Daisuke Nozawa, M.D., Ph.D., Toshinori Tsukanishi, M.D., Ph.D., Shigeo Izawa, M.D., and Akira Ikumi, M.D., Ph.D., who provided case illustrations 3, 4, 5 and 7, respectively.

\section{References}

1. Campana V, Milano G, Pagano E, et al.: Bone substitutes in orthopaedic surgery: from basic science to clinical practice. J Mater Sci: Mater Med. 2014, 25:2445-2461. 10.1007/s10856-014-5240-2

2. Urabe $\mathrm{K}$, Itoman $\mathrm{M}$, Toyama $\mathrm{Y}$, et al.: Current trends in bone grafting and the tissue of banked bone allografts based on the fourth nationwide survey of bone grafting status from 2000 to 2004. J Orthop Sci. 2007, 12:520-525. 10.1007/s00776-007-1174-6

3. Uchiyama K, Inoue G, Takahira N, Takaso M: Revision total hip arthroplasty-salvage procedures using bone allografts in Japan. J Orthop Sci. 2017, 22:593-600. 10.1016/j.jos.2017.01.023

4. Makihara T, Sakane M, Noguchi H, Yamazaki M: The balance between bone formation and material resorption in unidirectional porous $\beta$-tricalcium phosphate implanted in a rabbit tibia. Key Eng Mater. 2016, 696:177-182. 10.4028/www.scientific.net/KEM.696.177

5. Sakane M, Tsukanishi T, Funayama T, Kobayashi M, Ochiai N: Unidirectional porous $\beta$-Tricalcium phosphate bone substitute: examination of balance between new bone formation and absorption. Key Eng Mater. 2011, 493:132-134. 10.4028/www.scientific.net/KEM.493-494.132

6. Kumagai H, Makihara T, Funayama T, et al.: Angiogenesis and new bone formation in novel unidirectional porous beta-tricalcium phosphate: a histological study. J Artif Organs. 2019, 22:294-299. 10.1007/s10047019-01120-8

7. Ikumi A, Funayama T, Tsukanishi T, Noguchi H, Yamazaki M: Novel unidirectional porous $\beta$-Tricalcium phosphate used as a bone substitute after excision of benign bone tumors: a case series. J Hand Surg Asian Pac Vol. 2018, 23:424-429. https://doi.org/10.1142/S2424835518720293

8. Izawa S, Funayama T, Iwasashi M, Tsukanishi T, Kumagai H, Noguchi H, Yamazaki M: The use of unidirectional porous $\beta$-tricalcium phosphate in surgery for calcaneal fractures: a report of four cases. Foot 


\section{Cureus}

Ankle Online J. 2017 10,

9. Kumagai H, Abe T, Koda M, et al.: Unidirectional porous $\beta$-tricalcium phosphate induces bony fusion in lateral lumbar interbody fusion. J Clin Neurosci. 2019, 59:[Epub]. 10.1016/j.jocn.2018.09.004

10. Noguchi H, Funayama T, Koda M, et al.: A unidirectional porous beta-tricalcium phosphate material (Affinos ${ }^{\circledR}$ ) for reconstruction of bony defects after excision of fibular bone for spinal surgery graft. J Clin Neurosci. 2019, 66:71-76. 10.1016/j.jocn.2019.05.021

11. Kumagai H, Iwasashi M, Funayama T, Nakamura S, Noguchi H, Koda M, Yamazaki M: Surgical repair of acetabular fracture using unidirectional porous $\beta$-tricalcium phosphate. Case Rep Orthop. 2019, 2019:6860591. 10.1155/2019/6860591

12. Funayama $\mathrm{T}$, Tsukanishi $\mathrm{T}$, Kumagai $\mathrm{H}$, et al.: A novel unidirectional porous $\beta$-tricalcium phosphate grafting for vertebral fracture in the elderly: preliminary case series. J Rural Med. 2019, 14:211-215. $10.2185 / \mathrm{jrm} .3017$

13. Funayama T, Noguchi H, Mataki K, Yamazaki M: A novel unidirectional porous $\beta$-tricalcium phosphate grafting impregnated with bone marrow blood collected from unfractured vertebral bodies for non-union of osteoporotic vertebral fracture: a preliminary case report. J Orthop Case Rep. 2019, 9:50-53.

14. Kumagai H, Funayama $\mathrm{T}$, Sugaya $\mathrm{H}$, et al.: Effects of teriparatide on bone formation in novel unidirectional porous beta-tricalcium phosphate. J Biomater Appl. 2019, 34:721-727. 